\title{
Generation of cluster states
}

\author{
Ping Dong ${ }^{*}{ }^{1}$ Zheng-Yuan Xue, ${ }^{1}$ Ming Yang, ${ }^{1}$ and Zhuo-Liang Cao ${ }^{\dagger 1}$ \\ ${ }^{1}$ School of Physics \& Material Science, Anhui University, Hefei, 230039, P R China
}

\begin{abstract}
We propose two schemes for the generation of the cluster states. One is based on cavity quantum electrodynamics (QED) techniques. The scheme only requires resonant interactions between two atoms and a singlemode cavity. The interaction time is very short, which is important in view of decoherence. Furthermore, we also discuss the cavity decay and atomic spontaneous emission case. The other is based on atomic ensembles. The scheme has inherent fault tolerance function and is robust to realistic noise and imperfections. All the facilities used in our schemes are well within the current technology.
\end{abstract}

PACS numbers: 03.67.Hk, 03.65.Ud, 42.50.Dv

\section{INTRODUCTION}

In the realm of quantum information, entanglement is a universal resource. Some striking applications of entanglement have been proposed, such as quantum dense coding [1], quantum teleportation [2], quantum cryptography [3], etc. Generally, entangled states are used as a medium to transfer quantum information in quantum communication protocols. Moreover, they are used to speed up computation in quantum algorithms. While bipartite entanglement is well understood, multipartite entanglement is still under extensive exploration. For tripartite entangled quantum system, it falls into two classes of irreducible entanglement [4, 5, 6]. Recently, Briegel et al. [7] introduced a class of $N$-qubit entangled states, i.e., the cluster states, which have some special properties. The cluster states share the properties both of the GHZ- and of W-class entangled states. But they still have some unique properties, $e g$., they have a large persistency of entanglement, that is, they (in the case of $N>4$ ) are harder to be destroyed by local operations than GHZ-class states. In addition, they can be regarded as a resource for other multi-qubit entangled states. Thus the cluster states become an important resource in many branches of physics, especially in quantum information. Therefore, a number of applications using cluster states in quantum computation have been proposed [8, 9, 10, 11].

The generation of the cluster states attracted much attention. Recently Zou et al. proposed probabilistic schemes for generating the cluster states of four distant trapped atoms in leaky cavities [12], generating the cluster states in resonant microwave cavities [13] and generating the cluster states in linear optics system [14]. Barrett et al. proposed a protocol for generation of the cluster states using spatially separated matter qubits and single-photon interference effects [15] and so on [16, 17].

On the other hand, cavity quantum electrodynamics (QED) technique is a promising candidate for realizing the quantum processors. Meanwhile, much attention was paid to atomic ensembles in realizing the scalable long-distance quantum communication [18]. The schemes based on atomic en-

\footnotetext{
*pingdong@ahu.edu.cn

†zlcao@ahu.edu.cn(Corresponding Author)
}

sembles have some peculiar advantages compared with the schemes of quantum information processing by the control of single particles. Firstly, the schemes have inherent fault tolerance function and are robust to realistic noise and imperfections. Laser manipulation of atomic ensembles without separately addressing the individual atoms is dominantly easier than the coherent control of single particles. In addition, atomic ensembles with suitable level structure could have some kinds of collectively enhanced coupling to certain optical mode due to the multi-atom interference effects. Due to the above distinct advantages, a lot of novel schemes for the generation of quantum entangled states and quantum information processing have been proposed by using atomic ensembles [19, 20, 21, 22, 23]. Thus in this paper, we propose two schemes for the generation of the cluster states using cavity QED technique and the atomic ensembles. Our cavity QED scheme is different from that in Refs [12, 13]. The scheme only requires resonant interactions between two atoms and a single-mode cavity. The interaction time is very short, which is important in view of decoherence. More important, we consider the cavity decay and atomic spontaneous emission, which is unavoidable in the real process of generation. The proposal can be used to realize logic gates and directly transfer quantum information from one atom to another one [24] without using the cavity mode as the memory required in the previous experiment of Ref [25]. The scheme is very simple and can be generalized to the ion trap system. But for atomic ensembles scheme, as far as we known, this is the first scheme for the generation of the cluster states.

The paper is organized as follows: In section II , we introduce the cavity-QED model for generating a two-atom cluster state with and without cavity decay and atomic spontaneous emission, and then extend the scheme for two-atom cluster state to multi-atom cluster states case. Necessary discussions are also given in the end of the section. In section III, we discuss the scheme for generating the cluster states via atomic ensembles and then conclude the section and discuss the feasibility of our scheme. The conclusions appear in section IV. 


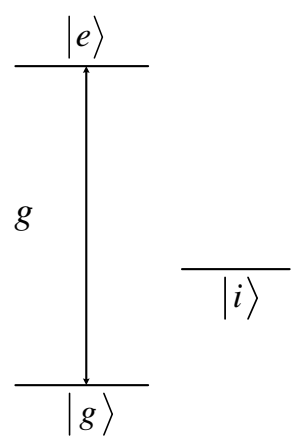

FIG. 1: The level structure of the atoms. $|g\rangle$ is the ground state, $|e\rangle$ is the excited state. The cavity mode is resonantly coupled to the $|e\rangle \leftrightarrow|g\rangle$ transition. The third level $|i\rangle$ is not affected by the interaction.

\section{GENERATION OF THE CLUSTER STATES WITH RESONANT INTERACTIONS}

In this section, we first use the resonant interaction between two atoms and a single-mode cavity to generate a twoatom cluster state. Three-level atoms are used in this model. The relevant atomic level structure is shown in Fig. 1 The third level $|i\rangle$ is not affected during the atom-cavity resonant interaction. Thus the Hamiltonian of the atom-cavity interaction can be expressed as, in the interaction picture (assuming $\hbar=1)[24]$

$$
H=g_{1}\left(a^{+} S_{1}^{-}+a S_{1}^{+}\right)+g_{2}\left(a^{+} S_{2}^{-}+a S_{2}^{+}\right),
$$

where $g_{1}$ and $g_{2}$ are the coupling strength of the atoms 1,2 with the cavity, respectively. $S^{+}=|e\rangle\left\langle g\left|, S^{-}=\right| g\right\rangle\langle e|$ and $|g\rangle$ is the ground state of the atoms, $|e\rangle$ is the excited state of the atoms. $a^{+}, a$ are the creation and annihilation operators for the cavity mode. Assume that the cavity mode is initially prepared in the vacuum state $|0\rangle$. In order to generate a two-atom cluster state, we prepare atom 1 in the state $|\phi\rangle_{1}=\frac{1}{\sqrt{2}}\left(|g\rangle_{1}+|e\rangle_{1}\right)$ and atom 2 in the state $|\phi\rangle_{2}=\frac{1}{\sqrt{2}}\left(|g\rangle_{2}+|i\rangle_{2}\right)$. So the initial state of the system is

$$
|\phi\rangle_{12 v}=\frac{1}{2}\left(|g\rangle_{1}+|e\rangle_{1}\right) \otimes\left(|g\rangle_{2}+|i\rangle_{2}\right) \otimes|0\rangle .
$$

Then we send the two atoms through the vacuum cavity, we can obtain the evolution [24]

$$
\begin{array}{r}
|e g\rangle_{12}|0\rangle \rightarrow \frac{g_{1}}{E}\left[\frac{1}{E}\left(g_{1} \cos (E t)+\frac{g_{2}^{2}}{g_{1}}\right)|e g\rangle_{12}|0\rangle\right. \\
\left.+\frac{1}{E} g_{2}[\cos (E t)-1]|g e\rangle_{12}|0\rangle-i \sin (E t)|g g\rangle_{12}|1\rangle\right], \\
|e i\rangle_{12}|0\rangle \rightarrow\left[\cos \left(g_{1} t\right)|e\rangle_{1}|0\rangle-i \sin \left(g_{1} t\right)|g\rangle_{1}|1\rangle\right]|i\rangle_{2}, \\
|g g\rangle_{12}|0\rangle \rightarrow|g g\rangle_{12}|0\rangle,
\end{array}
$$

$$
|g i\rangle_{12}|0\rangle \rightarrow|g i\rangle_{12}|0\rangle
$$

where $E=\sqrt{g_{1}^{2}+g_{2}^{2}}$. If we choose

$$
t=\frac{\pi}{g_{1}}, g_{2}=\sqrt{3} g_{1},
$$

which can be achieved by choosing coupling strengths and interaction time appropriately. Thus, we have

$$
|e g\rangle_{12}|0\rangle \rightarrow|e g\rangle_{12}|0\rangle,
$$

$$
|e i\rangle_{12}|0\rangle \rightarrow-|e i\rangle_{12}|0\rangle,
$$

$$
|g g\rangle_{12}|0\rangle \rightarrow|g g\rangle_{12}|0\rangle,
$$

$$
|g i\rangle_{12}|0\rangle \rightarrow|g i\rangle_{12}|0\rangle
$$

Then send atom 2 through a classical field tuned to the transition

$$
|i\rangle_{2} \rightarrow-|e\rangle_{2}
$$

These lead the state of atoms 1 and 2 to

$$
\begin{aligned}
|\phi\rangle_{12} & =\frac{1}{2}\left[|g\rangle_{1}\left(|g\rangle_{2}-|e\rangle_{2}\right)+|e\rangle_{1}\left(|g\rangle_{2}+|e\rangle_{2}\right)\right] \\
& =\frac{1}{2}\left(|g\rangle_{1} \sigma_{z}^{2}+|e\rangle_{1}\right)\left(|g\rangle_{2}+|e\rangle_{2}\right) .
\end{aligned}
$$

Obviously we get a standard two-atom cluster state. While in the real processing, the cavity decay and atomic spontaneous emission are unavoidable. Thus the discussion of these is necessary. Taking the cavity decay and atomic spontaneous emission into consideration, the Hamiltonian of the atom-cavity interaction can be expressed as (under the condition that no photon is detected either by the spontaneous emission or by the leakage of a photon through the cavity mirror and assuming $\hbar=1$ )

$$
\begin{aligned}
H & =g_{1}\left(a^{+} S_{1}^{-}+a S_{1}^{+}\right)+g_{2}\left(a^{+} S_{2}^{-}+a S_{2}^{+}\right) \\
& -i \frac{\kappa}{2} a^{+} a-i \frac{\tau}{2} \Sigma_{j=1}^{2}|e\rangle_{j}\langle e|,
\end{aligned}
$$

where $\kappa$ is the cavity decay rate and $\tau$ is the atomic spontaneous emission rate. If we send the atoms 1 and 2 through the vacuum cavity, choose the coupling strengths, interaction time $g_{2}=\sqrt{3} g_{1}, t=\frac{\pi}{g_{1}}$ appropriately and set $\kappa=\tau=0.1 g_{1}$, and then send the atom 2 through a classical field as in Eq. (6). Thus the state of the atoms 1 and 2 becomes

$$
\begin{array}{r}
|\phi\rangle_{12}=\sqrt{\frac{1}{2\left(1+e^{-\pi / 10}\right)}}\left[|g\rangle_{1}\left(|g\rangle_{2}-|e\rangle_{2}\right)\right. \\
\left.+e^{-\pi / 20}|e\rangle_{1}\left(|g\rangle_{2}+|e\rangle_{2}\right)\right] .
\end{array}
$$




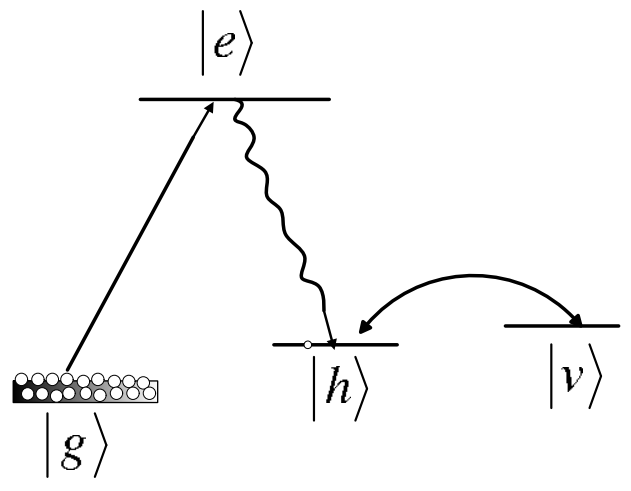

FIG. 2: The relevant atomic level structure of alkali metal atom. The transition of $|e\rangle \rightarrow|h\rangle$ can emit a forward-scattered Stokes photon co-propagating with the laser pulse. The excitation in the mode $h$ can be transferred to optical excitation by applying an anti-pump pulse.

The fidelity of this state relative to the standard two-atom cluster state in Eq. (7) is $\frac{\left(1+e^{-\pi / 20}\right)^{2}}{2\left(1+e^{-\pi / 10}\right)} \simeq 0.994$ and the probability of success is $\frac{1+e^{-\pi / 10}}{2} \simeq 0.865$. The fidelity and probability approach perfection.

Multi-atom entanglement is a very important source in quantum information processing and quantum computation. Especially the multi-atom cluster states attract many scientific attention recently, and some of their applications have been proposed [26, 27, 28, 29]. Thus the generation of multi-atom cluster states is vital for the construction of the practical quantum computers. Here, we generalize the above scheme of twoatom cluster state to multi-atom cluster states case.

We first prepare $N(N \geq 2)$ atoms in the states

$$
\begin{aligned}
& |\phi\rangle_{1}=\frac{1}{\sqrt{2}}\left(|g\rangle_{1}+|e\rangle_{1}\right), \\
& |\phi\rangle_{j}=\frac{1}{\sqrt{2}}\left(|g\rangle_{j}+|i\rangle_{j}\right),
\end{aligned}
$$

where $j=2,3 \cdots N$. The $N-1$ cavities are all prepared in vacuum states $|0\rangle$. So the total state of atoms is

$$
|\phi\rangle_{1 j}=\frac{1}{2^{N / 2}}\left(|g\rangle_{1}+|e\rangle_{1}\right) \bigotimes_{j=2}^{N}\left(|g\rangle_{j}+|i\rangle_{j}\right) .
$$

For the case of ideal cavity, firstly, we send atoms 1 and 2 through a vacuum cavity. The interaction between atoms 1, 2 and the cavity mode is governed by the Hamiltonian of Eq. 11. Meanwhile, we choose the coupling strengths, interaction time appropriately as in Eq. (4). Then send atom 2 through a classical field as in Eq. (6). These lead Eq. (11) to

$$
|\phi\rangle_{1 j}=\frac{1}{2^{N / 2}}\left(|g\rangle_{1} \sigma_{z}^{2}+|e\rangle_{1}\right)\left(|g\rangle_{2}+|e\rangle_{2}\right) \bigotimes_{j=3}^{N}\left(|g\rangle_{j}+|i\rangle_{j}\right) .
$$

Next, we send atoms 2 and 3 through another vacuum cavity. After the same interaction as on atoms 1 and 2, send atom 3 through a classical field as in Eq. [6, Here, Eq. (12) becomes

$$
\begin{array}{r}
|\phi\rangle_{1 j}=\frac{1}{2^{N / 2}}\left(|g\rangle_{1} \sigma_{z}^{2}+|e\rangle_{1}\right)\left(|g\rangle_{2} \sigma_{z}^{3}+|e\rangle_{2}\right) \\
\left(|g\rangle_{3}+|e\rangle_{3}\right) \bigotimes_{j=4}^{N}\left(|g\rangle_{j}+|i\rangle_{j}\right) .
\end{array}
$$

From the form of above states, we can conclude if we send two atoms through a vacuum cavity every time and then send one (the bigger subscript) of the two atoms through a classical filed, step by step, we can obtain the multi-atom cluster states easily. In other words, firstly we send atoms 1 and 2 through a vacuum cavity, then send atom 2 through a classical field. Secondly, we send atoms 2 and 3 through another vacuum cavity, then send atom 3 through another classical field, $\cdots$. Finally, we send atoms $N-1$ and $N$ through the last vacuum cavity, then send atom $N$ through a classical field. Thus the multi-atom cluster states can be obtained

$$
|\phi\rangle_{N}=\frac{1}{2^{N / 2}} \bigotimes_{j=1}^{N}\left(|g\rangle_{j} \sigma_{z}^{j+1}+|e\rangle_{j}\right)
$$

where $\sigma_{z}^{N+1} \equiv 1$.

For the case of real processing (with cavity decay and atomic spontaneous emission), we can obtain the cluster state by the same process as in the above ideal case and set $\kappa=$ $\tau=0.1 g_{1}$. We can obtain the cluster states

$$
\begin{aligned}
|\phi\rangle_{N} & =\sqrt{\frac{1}{2\left(1+e^{-\pi / 10}\right)^{N-1}}} \bigotimes_{j=1}^{N-1}\left(|g\rangle_{j} \sigma_{z}^{j+1}+e^{-\pi / 20}|e\rangle_{j}\right) \\
& \otimes\left(|g\rangle_{N} \sigma_{z}^{N+1}+|e\rangle_{N}\right) .
\end{aligned}
$$

While the fidelity of this state relative to the standard multiatom cluster state in Eq. (14) is $\left[\frac{\left(1+e^{-\pi / 20}\right)^{2}}{2\left(1+e^{-\pi / 10}\right)}\right]^{N-1}$ and the successful probability of obtaining the multi-atom cluster state is $\left(\frac{1+e^{-\pi / 10}}{2}\right)^{N-1}$. It is shown that the successful probability and fidelity both decrease exponentially with the increase of $N$.

Next, we briefly consider the feasibility of the current scheme. The scheme requires two atoms in a vacuum cavity, which have different coupling strengths with the cavity mode. The coupling depends on the atomic positions: $g=\Omega e^{-r^{2} / \omega^{2}}$, where $\Omega$ is the coupling strength at the cavity center, $\omega$ is the waist of the cavity mode, and $r$ is the distance between the atom and the cavity center [30]. The condition $g_{2}=\sqrt{3} g_{1}$ in our scheme can be satisfied by locating one atom at the center of the cavity and locating the other one at the position $r=\omega \sqrt{\ln \sqrt{3}}$. According to the recent experiments with Cs atoms trapped in an optical cavity [31], the condition can be obtained.

For the resonant cavity, in order to generate the cluster states successfully, the relationship between the interaction time and the excited atom lifetime should be taken into consideration. The interaction time should be much shorter than that of atom radiation. Hence, atom with a sufficiently long excited lifetime should be chosen. For Rydberg atom with 
principal quantum numbers 50 and 51 , the radiative time is $T_{1} \simeq 3 \times 10^{-2} \mathrm{~s}$. From the analysis in Ref [32], the interaction time is on the order $T \simeq 2 \times 10^{-4} \mathrm{~s}$, which is much shorter than the atomic radiative time. So the condition can be satisfied by choosing Rydberg atom. Our scheme requires that two atoms be simultaneously sent through a cavity, otherwise there will be an error. Assume that during the generation of a two-atom cluster state, one atom enters the cavity $0.01 t$ sooner than another atom, with $t$ being the time of each atom staying in the cavity. We can obtain the fidelity $F \simeq 0.999$ for generation of two-atom cluster state. Obviously in this case the operation is only slightly affected.

Furthermore one needs to reach the Lamb-Dicke regime in order to generate the cluster states successfully. For the initial state of Eq. (2), in the Lamb-Dicke regime, the infidelity caused by the spatial extension of the atomic wave function is about $\Delta \simeq(k a)^{2} \pi$, where $k$ is the wave vector of the cavity mode and $a$ is the spread of the atomic wave function. Setting $\Delta \simeq 0.01$, we have $a \simeq 0.01 \lambda$, where $\lambda$ is the wavelength of the cavity mode. If the atom trajectories cross the cavity with the deviation of less 0.1 degree from its pre-determined direction, we can ensure the fidelity is about 0.999 for generation of two-atom cluster state. While in order to maintain $g_{2}=\sqrt{3} g_{1}$ in the process of atomic motion in the cavity, we can choose the parameter of cavity $z \leq 0.5 z_{0}$, where $z_{0}=\frac{\pi \omega^{2}}{\lambda}$ and $2 z$ is the length of the cavity. We can obtain the error is only about $10^{-3}$. In the these cases, we can obtain the fidelity $F \simeq 0.999$ for generation of two-atom cluster state, which is bigger than the case of cavity decay and atomic spontaneous emission in the process of generation. Therefore our scheme is feasible with the current cavity QED technology.

The scheme for generating the cluster states in cavity QED only requires resonant interactions between two atoms and a single-cavity mode. The interaction time is very short, which is very important in view of decoherence. For the ideal case, the successful probability and the fidelity are both perfect (equal to 1.0). For the real case, the successful probability is 0.865 and the fidelity is 0.994 for the two-atom cluster states, while the successful probability and the fidelity for the multiatom cluster states both decrease exponentially with the increase of $N$. The scheme is very simple and can be generalized to the ion trap system.

\section{GENERATION OF THE CLUSTER STATES WITH ATOMIC ENSEMBLES}

In this section, we first introduce the basic system using in this paper. Atomic ensembles consist of a large number of identical alkali metal atoms. The relevant level structure of the alkali metal atoms is shown in Fig. 3 $|g\rangle$ is the ground state, $|e\rangle$ is the excited state and $|h\rangle,|v\rangle$ are two metastable states for storing a qubit of information, $e g$., Zeeman or hyperfine sublevels. For the three levels $|g\rangle,|h\rangle$ and $|v\rangle$, which can be coupled via a Raman process, two collective atomic operators can be defined as

$$
s=\left(1 / \sqrt{N_{a}}\right) \Sigma_{i=1}^{N_{a}}|g\rangle_{i}\langle s|,
$$

where $s=h, v$, and $N_{a} \gg 1$ is the total number of atoms. $s$ are similar to independent bosonic mode operators provided that all the atoms remain in ground state $|g\rangle$. The states of the atomic ensemble can be expressed as $|s\rangle=s^{+}|v a c\rangle$ $(s=h, v)$ after the emission of the single Stokes photon in a forward direction, where $|v a c\rangle \equiv \otimes_{i=1}^{N_{a}}|g\rangle_{i}$ denotes the ground state of the atomic ensemble.

It is necessary to discuss the realization of Controlled-Not gate for the generation of cluster states. The Controlled-Not gate can be realized via atomic ensembles with the help of Raman laser manipulations, beam splitters, and single-photon detections. Realization of Bell-basis measurement and generation of tripartite GHZ states is important for realization of Controlled-Not gate. Bell-basis measurement can be realized using the setup in Fig. 3 The four Bell states of the system are $|\phi\rangle_{A B}^{ \pm}=\left(h_{A}^{+} h_{B}^{+} \pm v_{A}^{+} v_{B}^{+}\right)|v a c\rangle_{A B} / \sqrt{2}$ and $|\varphi\rangle_{A B}^{ \pm}=$ $\left(h_{A}^{+} v_{B}^{+} \pm v_{A}^{+} h_{B}^{+}\right)|v a c\rangle_{A B} / \sqrt{2}$. We can use the setup to achieve the task, as shown in Fig. 3 Firstly, we apply anti-pump laser pulses to the two atomic ensembles $A$ and $B$ to transfer their $h$ excitations to optical excitations, and detect the anti-Stokes photons by detectors $D 1$ and $D 2$. If only detector $D 1$ (or $D 2$ ) clicks, we will apply single-qubit rotations to both ensembles to rotate their $v$ modes to $h$ modes by shinning $\pi$ length $\mathrm{Ra}$ man pulses or radio-frequency pulses on the two ensembles $A$ and $B$. Then we apply anti-pump laser pulses to two atomic ensembles $A$ and $B$ again, and detect anti-Stokes photons by $D 1$ and $D 2$. Now, there are two different results of detection: (1) If detector $D 1$ (or $D 2$ ) clicks (one detector clicks twice in the two detections), post-select the cases that each ensemble has only one excitation, atomic ensembles $A$ and $B$ are projected into $|\varphi\rangle_{A B}^{+}=\left(h_{A}^{+} v_{B}^{+}+v_{A}^{+} h_{B}^{+}\right)|v a c\rangle_{A B} / \sqrt{2}$; (2)If D2 (or $D 1$ ) clicks (detectors $D 1$ and $D 2$ click respectively in the two detections), post-select the cases that each ensemble has only one excitation, atomic ensembles $A$ and $B$ are projected into $|\varphi\rangle_{A B}^{-}=\left(h_{A}^{+} v_{B}^{+}-v_{A}^{+} h_{B}^{+}\right)|v a c\rangle_{A B} / \sqrt{2}$. Obviously, if we add single-qubit rotations in the above process, we can realize the projection of $|\phi\rangle_{A B}^{ \pm}=\left(h_{A}^{+} h_{B}^{+} \pm v_{A}^{+} v_{B}^{+}\right)|v a c\rangle_{A B} / \sqrt{2}$ by post-selecting sense.

Tripartite GHZ states can be prepared using the protocol of Ref [19] with atomic ensembles. Firstly, atomic ensembles 1 and 2 can be prepared in the state $|\phi\rangle_{12}^{ \pm}=\left(h_{1}^{+} \pm\right.$ $\left.e^{i \varphi} h_{2}^{+}\right)|v a c\rangle_{12} / \sqrt{2}$ as in Ref [18]. Then we can omit $e^{i \varphi}$ by the way in [19] and perform a single-qubit rotation on atomic ensemble 2 . The state of atomic ensembles 1 and 2 becomes $|\phi\rangle_{12}=\left(h_{1}^{+}+v_{2}^{+}\right)|v a c\rangle_{12} / \sqrt{2}$. Secondly, we prepare the atomic ensembles 2, 3 and 3,1 in the states $|\phi\rangle_{23}=$ $\left(h_{2}^{+}+v_{3}^{+}\right)|v a c\rangle_{23} / \sqrt{2}$ and $|\phi\rangle_{31}=\left(h_{3}^{+}+v_{1}^{+}\right)|v a c\rangle_{31} / \sqrt{2}$. So the total state becomes $|\phi\rangle_{123}=|\phi\rangle_{12} \otimes|\phi\rangle_{23} \otimes|\phi\rangle_{31}$. Post-select the case that each ensemble has only one excitation, we can obtain the GHZ state $|\phi\rangle_{123}=\left(h_{1}^{+} h_{2}^{+} h_{3}^{+}+\right.$ $\left.v_{1}^{+} v_{2}^{+} v_{3}^{+}\right)|v a c\rangle_{123} / \sqrt{2}$. In the same way, we can prepare another GHZ state using atomic ensembles 4, 5 and $6|\phi\rangle_{456}=$ $\left(h_{4}^{+} h_{5}^{+} h_{6}^{+}+v_{4}^{+} v_{5}^{+} v_{6}^{+}\right)|v a c\rangle_{456} / \sqrt{2}$.

In order to realize C-NOT gate, we prepare two atomic ensembles 7 and 8 ( ensemble 7 as control, ensemble 8 as target), which are in $|\phi\rangle_{7}=\left(h_{7}^{+}+v_{7}^{+}\right)|v a c\rangle_{7}$ and $|\phi\rangle_{8}=$ $\left(h_{8}^{+}-v_{8}^{+}\right)|v a c\rangle_{8}$ by single-qubit rotations. Firstly, we apply Hadamard transformations on atomic ensembles 1, 2 and 3 re- 


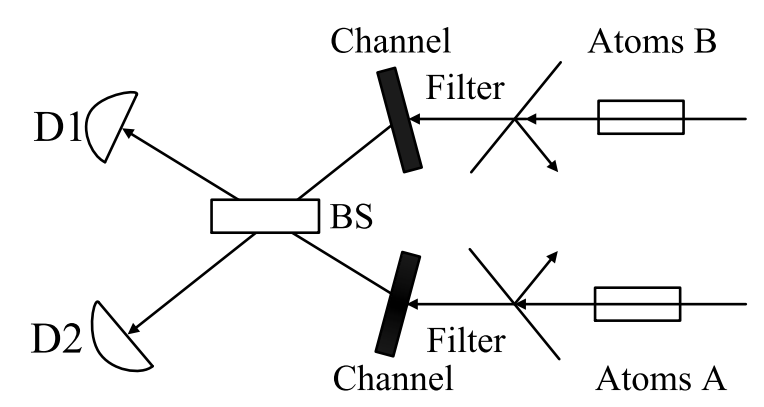

FIG. 3: Setup of realizing Bell-basis measurement. The two atomic ensembles A and B are pencil-shaped, which are illuminated by the synchronized laser pulses. The forward-scattered anti-Stokes photons are collected and coupled to optical channel (fiber) after the filter. BS is a 50/50 beam splitter, and the outputs are detected by two single-photon detectors $D 1$ and $D 2$.

spectively, and then make a Bell-basis measurement on atomic ensembles 3 and 4 . Then the state $|\phi\rangle_{123456}$ collapses to one of the following four unnormalized states

$$
\begin{aligned}
|\phi\rangle_{1256} & =\left[\left(h_{1}^{+} h_{2}^{+}+v_{1}^{+} v_{2}^{+}\right) h_{5}^{+} h_{6}^{+}\right. \\
& \left. \pm\left(h_{1}^{+} v_{2}^{+}+v_{1}^{+} h_{2}^{+}\right) v_{5}^{+} v_{6}^{+}\right]|v a c\rangle_{1256}, \\
|\varphi\rangle_{1256} & =\left[\left(h_{1}^{+} h_{2}^{+}+v_{1}^{+} v_{2}^{+}\right) v_{5}^{+} v_{6}^{+}\right. \\
& \left. \pm\left(h_{1}^{+} v_{2}^{+}+v_{1}^{+} h_{2}^{+}\right) h_{5}^{+} h_{6}^{+}\right]|v a c\rangle_{1256} .
\end{aligned}
$$

Where $|\phi\rangle_{1256}$ and $|\varphi\rangle_{1256}$ are the results of the projection into $|\phi\rangle_{34}^{ \pm}$and $|\varphi\rangle_{34}^{ \pm}$, respectively. They can unify as $|\chi\rangle_{1256}=\left[\left(h_{1}^{+} h_{2}^{+}+v_{1}^{+} v_{2}^{+}\right) h_{5}^{+} h_{6}^{+}+\left(h_{1}^{+} v_{2}^{+}+\right.\right.$ $\left.\left.v_{1}^{+} h_{2}^{+}\right) v_{5}^{+} v_{6}^{+}\right]|v a c\rangle_{1256}$ with the help of simple single-qubit operations.

Then we make Bell-basis measurements on atomic ensembles 1,8 and 6,7 . The state of atomic ensembles 2 and 5 collapses to one of the following states

$$
\begin{aligned}
& |\phi\rangle_{25}=\left(h_{2}^{+}-v_{2}^{+}\right)\left(h_{5}^{+}-v_{5}^{+}\right)|v a c\rangle_{25} / 2, \\
& |\varphi\rangle_{25}=\left(h_{2}^{+}-v_{2}^{+}\right)\left(h_{5}^{+}+v_{5}^{+}\right)|v a c\rangle_{25} / 2 .
\end{aligned}
$$

where Eq. 17a corresponds to the measurement results of $|\phi\rangle_{67}^{+}$and $|\varphi\rangle_{67}^{+}$, and Eq. $\left.117 \mathrm{~b}\right\rangle$ corresponds to $|\phi\rangle_{67}^{-}$and $|\varphi\rangle_{67}^{-}$. We can transform state 17b to state 17a by single-qubit rotations. Obviously, C-NOT gate has been realized and the state of atomic ensembles 7 and 8 has been mapped on ensembles 2 and 5 .

Next, we discuss the generation of bipartite cluster state. The atomic ensembles 1 and 2 are initially prepared in the state

$$
|\phi\rangle_{12}=v_{1}^{+} v_{2}^{+}|v a c\rangle_{12}
$$

using Raman pulses. All the single-qubit transformation can be achieved by laser pulses in atomic ensembles. Secondly, we perform a single-qubit operation on atomic ensemble 1

$$
v_{1}^{+}|v a c\rangle_{1} \rightarrow\left(h_{1}^{+}+v_{1}^{+}\right)|v a c\rangle_{1} / \sqrt{2}
$$

Then, we perform a Controlled-Not transformation on the two atomic ensembles, where atomic ensemble 1 serving as control qubit and atomic ensemble 2 as target qubit. Now, the above procedures lead Eq. 18 to

$$
|\phi\rangle_{12}=\left(h_{1}^{+} v_{2}^{+}+v_{1}^{+} h_{2}^{+}\right)|v a c\rangle_{12} / \sqrt{2} .
$$

Finally, we perform a single-qubit operation on atomic ensemble 1

$$
h_{1}^{+}|v a c\rangle_{1} \rightarrow v_{1}^{+}|v a c\rangle_{1}, v_{1}^{+}|v a c\rangle_{1} \rightarrow h_{1}^{+}|v a c\rangle_{1},
$$

and another single-qubit operation on atomic ensemble 2

$$
\begin{aligned}
h_{2}^{+}|v a c\rangle_{2} & \rightarrow\left(h_{2}^{+}-v_{2}^{+}\right)|v a c\rangle_{2} / \sqrt{2}, \\
v_{2}^{+}|v a c\rangle_{2} & \rightarrow\left(h_{2}^{+}+v_{2}^{+}\right)|v a c\rangle_{2} / \sqrt{2}
\end{aligned}
$$

Here, the quantum state of atomic ensembles 1 and 2 becomes

$$
\begin{aligned}
|\phi\rangle_{12} & =\left[h_{1}^{+}\left(h_{2}^{+}-v_{2}^{+}\right)+v_{1}^{+}\left(h_{2}^{+}+v_{2}^{+}\right)\right]|v a c\rangle_{12} / 2 \\
& =\left[\left(h_{1}^{+} \sigma_{z}^{2}+v_{1}^{+}\right)\left(h_{2}^{+}+v_{2}^{+}\right)\right]|v a c\rangle_{12} / 2 .
\end{aligned}
$$

Obviously the state is a standard bipartite cluster states $(N=2)$. The cluster states $(N=2,3)$ can be also generated without Controlled-Not transformation [18, 19]. However, for the generation of the multipartite cluster states, using the proposals of Ref. [18, 19] are very hard, while, it can be realized by the above method with Controlled-Not transformations, as shown below.

Out of question, for the generation of arbitrary $N$-particle cluster state $(N \geq 2)$, we can use the single-qubit operations and controlled-not transformations to achieve the task perfectly. Here, we discuss the process in detail. Firstly, we prepare $N$ atomic ensembles, which are all in the states $v_{i}^{+}|v a c\rangle_{i}$ $(i=1,2 \cdots N)$. So the state of the whole system is

$$
|\phi\rangle_{12 \cdots N}=\left(v_{1}^{+} v_{2}^{+} \cdots v_{N}^{+}\right)|v a c\rangle_{12 \cdots N} .
$$

Secondly, we perform appropriately transformations as the above process on atomic ensembles 1 and 2 (Eq. (19)-(22), which lead the initial state to

$$
\begin{array}{r}
|\phi\rangle_{12 \cdots N}=\left(h_{1}^{+} \sigma_{z}^{2}+v_{1}^{+}\right)\left(h_{2}^{+}+v_{2}^{+}\right) \\
\left(v_{3}^{+} v_{4}^{+} \cdots v_{N}^{+}\right)|v a c\rangle_{12 \cdots N} / 2 .
\end{array}
$$

Then, we perform the same transformations on atomic ensembles 2 and 3 as atomic ensembles 1 and 2. We can obtain the result

$$
\begin{array}{r}
|\phi\rangle_{12 \cdots N}=\left(h_{1}^{+} \sigma_{z}^{2}+v_{1}^{+}\right)\left(h_{2}^{+} \sigma_{z}^{3}+v_{2}^{+}\right)\left(h_{3}^{+}+v_{3}^{+}\right) \\
\left(v_{4}^{+} v_{5}^{+} \cdots v_{N}^{+}\right)|v a c\rangle_{12 \cdots N} / 2 \sqrt{2}
\end{array}
$$

In a word, if we perform the transformations of Eq. 19222 on atomic ensembles 1 and 2, then on atomic ensembles 2 and 3, up to on atomic ensembles $N-1$ and $N$, we will obtain the perfect multipartite cluster states 


$$
\begin{aligned}
|\phi\rangle_{12 \cdots N} & =\frac{1}{2^{N / 2}}\left(h_{1}^{+} \sigma_{z}^{2}+v_{1}^{+}\right)\left(h_{2}^{+} \sigma_{z}^{3}+v_{2}^{+}\right) \\
& \cdots\left(h_{N}^{+}+v_{N}^{+}\right)|v a c\rangle_{12 \cdots N} \\
& =\frac{1}{2^{N / 2}} \bigotimes_{i=1}^{N}\left(h_{i}^{+} \sigma_{z}^{i+1}+v_{i}^{+}\right)|v a c\rangle_{12 \cdots i}
\end{aligned}
$$

where $\sigma_{z}^{N+1} \equiv 1$.

We briefly discuss the feasibility of the current scheme. If we want to generate a high-fidelity entangled state about 16 ensembles, a time $T_{i m p} \simeq 50 \mathrm{~ms}$ will be needed by choosing other parameters appropriately, which has been proved [19]. With such a short preparation time $T_{i m p}$, the noise that we have not included is negligible, such as the nonstationary phase drift induced by the pumping phase or by the optical channel. As long as the number $n$ of the ensembles is not huge, we also can safely neglect the single-bit rotation error ( below $10^{-4}$ with the use of accurate polarization techniques for Zeeman sublevels [33] ) and the dark count probability of single-photon detectors (about $10^{-5}$ in a typical detection time window $0.1 \mu s$ [19]). Thus it seems reasonable to generate cluster states over tens of ensembles with the current technology. Furthermore, the scaling can be made polynomial by dividing the whole preparation process into small steps, checking in each steps and repeating these steps instead of the whole process in case it fails. So our scheme has inherent fault tolerance function and is robust to realistic noise and imperfections [18, 19].

The physical scheme for generating the cluster states based on atomic ensembles have some peculiar advantages compared with the schemes by the control of single particles, $e g$. the schemes have inherent fault tolerance function and are robust to realistic noise and imperfections. Laser manipulation of atomic ensembles without separately addressing the individual atoms is dominantly easier than the coherent control of single particles. Atomic ensembles with suitable level structure could have some kinds of collectively enhanced coupling to certain optical mode due to the multi-atom interference effects and so on [18]. At the same time, We can generate the $\mathrm{N}$-qubit cluster state simply by extending the two-qubit case.

\section{CONCLUSIONS}

We propose two schemes for the generation of the cluster states. One scheme is based on cavity quantum electrodynamics (QED) technics. The scheme only requires resonant interactions between two atoms and a single-mode cavity. The interaction time is very short, which is important in view of decoherence. We first introduce the two-atom case then extend it to mult-atom case. Furthermore, we consider the cavity decay and atomic spontaneous emission case, the successful probability and the fidelity for the multi-atom cluster states both decrease exponentially with the increase of $N$. The scheme is very simple and can be generalized to the ion trap system. The other is based on atomic ensembles. The scheme has inherent fault tolerance function and is robust to realistic noise and imperfections. The generation of cluster states from twoqubit case to multi-qubit case is simple and feasible. All of the facilities used in our schemes are well within the current technology.

\section{Acknowledgments}

This work is supported by the Natural Science Foundation of the Education Department of Anhui Province under Grant No: 2006kj070A and Anhui Provincial Natural Science Foundation under Grant No: 03042401 and the Talent Foundation of Anhui University.
[1] C. H. Bennett and S. J. Wiesner, Phys. Rev. Lett. 69, 2881 (1992).

[2] C. H. Bennett, G. Brassard, C. Crépeau, R. Jozsa, A. Peres and W. K. Wootters, Phys. Rev. Lett. 70, 1895 (1993).

[3] A. K. Ekert, Phys. Rev. Lett. 67, 661 (1991).

[4] M. Greenberger, M. A. Horne and A. Zeilinger, Am. J. Phys. 58, 1131 (1990).

[5] W. Dür, G. Vidal and J.I. Cirac, Phys. Rev. A 62, 062314 (2000).

[6] A. Acín, D. Bruß, M. Lewenstein and A. Sanpera, Phys. Rev. Lett. 87, 040401 (2001).

[7] H. J. Briegel and R. Raussendorf, Phys. Rev. Lett. 86, 910 (2001).

[8] D. L. Zhou, B. Zeng, Z. Xu, and C. P. Sun, Phys. Rev. A. 68, 062303 (2003).

[9] R. Raussendorf, D. E. Browne, and H. J. Briegel,Phys. Rev. A. 68, 022312 (2003).

[10] M. A. Nielsen, Phys. Rev. Lett. 93, 040503 (2004).

[11] M. A. Nielsen and C. M. Dawson, Phys. Rev. A. 71, 042323
(2005).

[12] X. B. Zou, K. Pahlke, and W. Mathis, Phys. Rev. A 69, 052314 (2004).

[13] X. B. Zou and W. Mathis, Phys. Rev. A 71, 032308 (2005).

[14] X. B. Zou and W. Mathis, Phys. Rev. A 72, 013809 (2005).

[15] S. D. Barrett and P. Kok, Phys. Rev. A. 71, 060310(R) (2005).

[16] M. Borhani and D. Loss, Phys. Rev. A. 71, 034308 (2005).

[17] J. Cho and H. W. Lee, Phys. Rev. Lett. 95, 160501 (2005).

[18] L. M. Duan, M. D. Lukin, J. I. Cirac and P. Zoller, Nature 414, 413 (2001).

[19] L. M. Duan, Phys. Rev. Lett. 88, 170402 (2002).

[20] L. M. Duan, J. I. Cirac and P. Zoller, Phys. Rev. A. 66, 023818 (2002).

[21] P. Xue and G. C. Guo, Phys. Rev. A. 67, 034302 (2003).

[22] M. D. Lukin, S. F. Yelin and M. Fleischhauer, Phys. Rev. Lett. 84, 4232 (2000).

[23] C. Liu, Z. Dutton, C. H. Behroozi, L. V. Hau, Nature 409, 490 (2001).

[24] S. B. Zheng, Phys. Rev. A. 71, 062335 (2005). 
[25] X. Maitre, E. Hagley, G. Nogues, C. Wunderlich, P. Goy, M. Brune, J. M. Raimond, and S. Haroche, Phys. Rev. Lett. 79,769 (1997).

[26] M. S. Tame, M. Paternostro, M. S. Kim, and V. Vedral, Phys. Rev. A. 72, 012319 (2005).

[27] D. L. Zhou, B. Zeng, Z. Xu, and C. P. Sun, Phys. Rev. A. 68, 062303 (2003).

[28] R. Raussendorf, D. E. Browne, and H. J. Briegel, Phys. Rev. A. 68, 022312 (2003).

[29] M. A. Nielsen and C. M. Dawson, Phys. Rev. A. 71, 042323,
(2005).

[30] S. Osnaghi, P. Bertet, A. Auffeves, P. Maioli, M. Brune, J. M. Raimond, and S. Haroche, Phys. Rev. Lett. 87, 037902 (2001).

[31] A. Boca, R. Miller, K.M. Birnbaum, A. D. Boozer, J. Mckeever, and H. J. Kimble, Phys. Rev. Lett. 93, 233603 (2004).

[32] S. B. Zheng and G. C. Guo, Phys. Rev. Lett. 85, 2392 (2000).

[33] D. Budker, V. Yashchuk, and M. Zolotorev, Phys. Rev. Lett. 81, 5788 (1998). 